\title{
Calidad de Vida y Factores Biopsicosociales en Patologías Médicas Crónicas ${ }^{1}$
}

\section{Quality of Life and Biopsychosocial Factors in Chronic Medical Phatologies}

\author{
Alfonso Urzúa M. \\ Universidad Católica del Norte, Chile \\ (Rec: 20 septiembre, 2008 Acep: 04 noviembre 2008)
}

\begin{abstract}
Resumen
Con base en un diseño transversal, se evaluó la calidad de vida y su relación con factores biopsicosociales en 240 participantes, 120 de los cuales vivían con una patología médica crónica prevalente en Atención Primaria de Salud. El principal instrumento utilizado fue el WHOQoL-Bref de la OMS. Se encuentran como variables relacionados significativamente con la calidad de vida la edad, la satisfacción con la salud, el afecto positivo, la balanza de afectos, la satisfacción con la vida y la efectividad de la medicación y de manera inversa con el afecto negativo. No se encuentra relación entre la calidad de vida y el sexo, el apoyo social objetivo y el tiempo viviendo con la enfermedad.
\end{abstract}

Palabras clave: Calidad de vida, factores biopsicosociales, enfermedad crónica.

\begin{abstract}
Based on a transectional design, quality of life and its relationship to biopsychosocial factors was evaluated in 240 participants, 120 of whom where living with a chronic medical pathology of Primary Health Attention. The main instrument used was the WHOQoL- Bref of the WHO. Satisfaction with health, positive affect, the affect balance, satisfaction with life, and mediation efficiency, were positively related to quality of life; negative affect was inversely correlated to quality of life. There was no correlation of quality of life with sex, social support, and time with the illness.
\end{abstract}

Key words: Quality of life, chronic illness, biopsychosocial variables. 


\section{Introducción}

La Calidad de vida (CV) puede ser definida como el estado o sentimiento de bienestar derivado de la evaluación tanto objetiva como subjetiva del grado de satisfacción de la persona en distintas dimensiones de su vida.

Los estudios realizados en esta área no son concluyentes, ya que aún cuando la gran mayoría de los investigadores reporta la presencia de una enfermedad crónica como un elemento modulador de la $\mathrm{CV}$, algunos autores sugieren que no existirían diferencias en la $\mathrm{CV}$ entre crónicos y no crónicos (Cassileth et al., 1984), o bien que existirían diferencias dada la condición o tipo de cronicidad (Kempen, Jelicic \& Ormel, 1997; Alonso et al., 2004; Sprangers, M., de Regt, E., Andries, F., et al. 2000; Stavem, Lossius, Kvien \& Guldvog (2001)

De igual manera, existe inconsistencia en la vinculación que se ha hecho de algunas variables psicológicas y sociales con la $\mathrm{CV}$ en personas con patologías crónicas no transmisibles, observándose algunas variables que han sido encontradas en mayor o menor medida asociadas a la CV tales como: gravedad de la enfermedad o severidad de los síntomas (Croyle, Troester, Fields, Straits, Lyons \& Pahwa, 2003 ; Frazier, 2000; Luscombe, 2000; Dalfó, Badía, Roca, Aristegui \& Roset, 2000; Meszaros, Zelkó, Meskó \& Vincze, 2005.), percepción de control (Croyle et al 2003; Carroll, 1999; Hart, Bilo, Redekop, Stolk, Assink \& Meyboom de Jong, 2003; Burckhardt, 1985, en Michalos et al., 2000 ; Pucheu et al., 2004), edad (Pucheu et al., 2004, Meszaros et al 2005 ; Frazier, 2000), sexo (Frazier, 2000; Hart et al 2003), apoyo social (Ethgen et al., 2004; Bennet et al. 2001; Han, Lee \& Park, 2003 ; Pucheu et al., 2004).

En este marco, esta investigación tuvo por objeto estudiar la relación existente entre la presencia de una enfermedad crónica y la $\mathrm{CV}$, así como la relación de esta última con factores psicológicos y sociales mencionados frecuentemente en la literatura y que afectarían la $\mathrm{CV}$ de las personas que viven con patologías médicas crónicas prevalentes.

Se escoge la Atención Primaria en Salud dada su condición de puerta de entrada al sistema sanitario y al alto número de pacientes con enfermedades crónicas que utilizan el sistema público de atención en salud. Se opta además por utilizar un instrumento construido por la Organización Mundial de la Salud, que permite ser usado en diferentes grupos con distintos niveles y que permitan aportar a la comparación con poblaciones similares en otras partes del mundo en la investigación sobre qué factores modulan la percepción de la calidad de vida de las personas que viven con una patología crónica.

\section{Método}

\section{Participantes}

La muestra total estuvo conformada por 240 participantes, de los cuales 120 vivían con una patología médica crónica. Las muestras fueron pareadas, por lo que ambos sexos presentan igual porcentaje de representación. La media de edad para ambos grupos correspondió a 53,5 años (d.e. 11,2 años).

Los participantes fueron elegidos en forma aleatoria entre todas aquellas personas que acudían al control de crónicos de médico o enfermera de los diversos Consultorios, siendo encuestadas 13 personas con trastornos músculo esqueléticos (artritis y artrosis), 58 personas con hipertensión, 33 personas con diabetes y 16 con trastornos crónicos varios (dolor crónico, epilepsia, cáncer). El tiempo promedio viviendo con la enfermedad correspondió a 7 años. (d.e. $=7,4$ años).

Para la inclusión y/o exclusión se consideraron como criterios: ser mayor de 18 años, no padecer problemas mentales y/o del Sistema Nervioso Central que dificulten la capacidad de comprensión de las encuestas y acceder voluntariamente a participar en la encuesta; para ello se obtuvo el consentimiento oral informado de cada persona.

\section{Procedimientos}

La muestra de crónicos se obtuvo en Consultorios de Atención Primaria de la ciudad de Antofagasta y en grupos de adultos con patología crónica dependientes de los mismos Consultorios. Los controles fueron obtenidos de familiares de los pacientes crónicos, de acompañantes de las personas a los controles de salud y en grupos organizados de adultos mayores de la ciudad.

Los cuestionarios fueron autoadministrados y en aquellos casos que presentaron dificultad para leer el instrumento, estos fueron contestando las preguntas al entrevistador, apoyadas con un sistema de tarjetas con las alternativas de respuesta a cada pregunta. El tiempo promedio de cada encuesta fue de 45 minutos.

Una vez obtenidas todas las encuestas, estas fueron ingresadas a una base de datos en el software SPSS 11.5 para su posterior análisis.

\section{Instrumentos}

Para evaluar la CV se utilizó el cuestionario WHOQoLBREF, de la Organización Mundial de la Salud, cuya elaboración y propiedades psicométricas en diversas poblaciones han sido reportadas en diversas investigaciones (Skevington, Lotfy \& O'Conell, 2004; The WHOQOL Group, 1998, Barros da Silva et al., 2005; Jang et al., 2004, Saxena et al., 2001). 
EL WHOQoL-BREF consta de 26 reactivos agrupados en cuatro factores: físico, psicológico, social y ambiental, más una pregunta sobre percepción de $\mathrm{CV}$ general y otra sobre satisfacción con la salud.

Sobre la validación de este instrumento en el idioma castellano solo se encuentran referencias del trabajo de Lucas (1998). En el presente estudio, la consistencia interna (medida igualmente a través del alfa de Cronbach) fue de 0,69 para el dominio físico, 0,74 para el psicológico, 0,77 para el ambiental y un 0,70 para el dominio de las relaciones sociales.

La Balanza de afectos fue evaluada a través de la escala de Bradburn (1969) y tiene por objeto medir el equilibrio afectivo de los individuos durante el último mes. Dicho instrumento consta de dos subescalas, una sobre afecto positivo y otra sobre el negativo.

La percepción de control de la enfermedad fue evaluada a través de tres preguntas: una pregunta elaborada con base a la propuesta del locus de control en salud realizada por Wallston y Wallston (1999), que posibilita el ubicar a la persona en tres tipos de categorías de locus dependiendo del grado de creencia en la persona de que los factores internos, externos o la suerte son los responsables de su propia salud o enfermedad.

La segunda pregunta es sobre el grado de efecto percibido de la medicación sobre los síntomas de la enfermedad, considerando que un mejor efecto de la medicación pudiese dar una mayor sensación de control sobre ésta. Comprende las categorías muy efectiva, más o menos efectiva, poco efectiva e inefectiva.

La tercera pregunta es acerca de la percepción del sujeto acerca del grado de control que él percibe tiene sobre su propia enfermedad, pudiendo optar por siempre, frecuentemente, rara vez y nunca.

El apoyo social es medido a través de la escala adaptada de Vaux et al., 1986. Consta de una subescala destinada a medir el apoyo social subjetivo, y otra para evaluar el apoyo social objetivo.

\section{Análisis de datos}

Se realizó primeramente la descripción de las variables en su relación con la calidad de vida y posteriormente las pruebas de relación entre estas. Dependiendo del tipo de variables se utilizaron las pruebas U de Mann-Whitney y Rho de Spearman,

\section{Resultados}

\section{Calidad de Vida General}

En términos generales, el mayor porcentaje de los encuestados, tanto a nivel de crónicos y control, declaran tener una CV "normal", oscilando desde un $50 \%$ entre los crónicos a casi un $65 \%$ en los no crónicos. Un $24,2 \%$ de los crónicos declaran no tener una buena calidad de vida, en contraposición a solo un 2,5\% de los no crónicos. Estas diferencias, evaluadas a través del estadístico U de MannWhitney, son estadísticamente significativas, siendo, al comparar los rangos promedios de los grupos crónicos y de controles, significativamente mayor el rango promedio en $\mathrm{CV}$ en la muestra de controles sobre la muestra de crónicos $(\mathrm{z}=4,097 ; \mathrm{p}<0,001)$.

\section{Calidad de Vida y Variables Sociodemográficas}

$\mathrm{Al}$ analizar la relación entre la edad y la CV percibida, a través del estadístico rho de Spearman, se puede observar que esta es significativa solamente a nivel de la muestra de crónicos, de manera inversa y con una muy baja intensidad de la relación $\left(\mathrm{r}=-0,227 ; \mathrm{p}=0,013 ; \mathrm{r}^{2}=0,052\right)$.

Con relación al sexo de los participantes, al analizar a través de la prueba U de Mann Whitney, la distribución porcentual de respuestas en las diversas categorías de evaluación de la $\mathrm{CV}$ no se encuentran diferencias significativas entre los rangos promedios para el total de hombres y el total de mujeres.

\section{Calidad de Vida General y Dominios de Calidad de Vida}

$\mathrm{Al}$ analizar la relación existente entre la $\mathrm{CV}$ general y los distintos dominios que evalúa el WHOQoL- BREF, se encuentra que tanto a nivel de la muestra en su totalidad, como separando por condición de salud, la relación es estadísticamente significativa y directa. La medida del tamaño del efecto muestra una baja intensidad en todas las relaciones.

\section{Calidad de Vida y Satisfacción con la vida y con la salud}

Se encuentra que la variable satisfacción con la vida está correlacionada con la $\mathrm{CV}$ en todos los niveles de análisis, tanto a nivel general, como por condición, siendo la relación en todos los casos de tipo directo y con una medida del tamaño del efecto que indica una baja intensidad de la relación.

Asimismo, la relación entre CV general y satisfacción con la salud es estadísticamente significativa en todas las instancias: a nivel general la medida del tamaño del efecto indica una moderada intensidad de la relación $(\mathrm{r}=0,613$; $\mathrm{p}<0,001 ; \mathrm{r}^{2}=0,376$ ), de igual manera para crónicos y no crónicos $\left(r=0,653 ; p<0,001 ; r^{2}=0,426\right)$

\section{Calidad de Vida y Locus de control}

$\mathrm{Al}$ analizar la relación existente entre Locus de control, categorizado como externo e interno, y la $\mathrm{CV}$, a través de la prueba no paramétrica U de Mann Whitney, se encuentra 
Tabla 1.

Relación Apoyo Social Objetivo y Subjetivo y Calidad de Vida General por condición

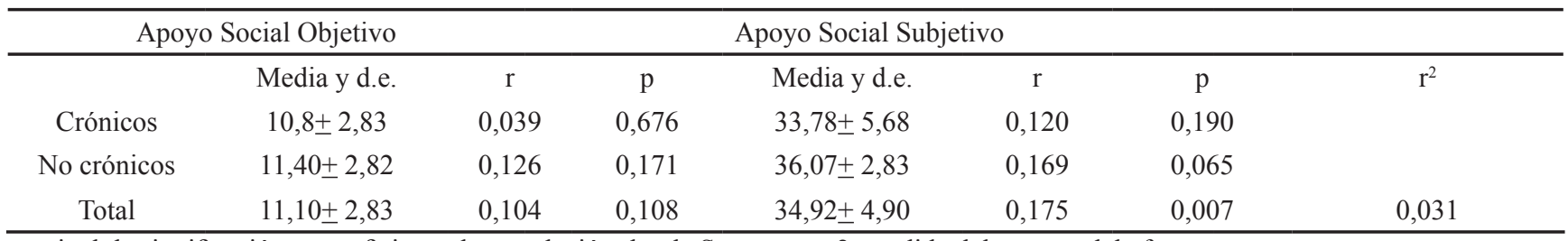

$\mathrm{p}=$ nivel de significación; r: coeficiente de correlación rho de Spearman; r2: medida del tamaño del efecto

que a nivel de la muestra total, la media del rango en $\mathrm{CV}$ es significativamente mayor en aquellos que tienen un locus de control interno por sobre aquellos en que predomina un externo $(z=2,724 ; p=0,006)$. Sin embargo, al estratificar el análisis por condición de morbilidad se encuentra que esta significación se mantiene solo a nivel de la población de crónicos $(\mathrm{z}=-2,191 ; \mathrm{p}=0,028)$ en donde la media del rango en $\mathrm{CV}$ es superior en aquellos que tienen un locus interno, no siendo así en la muestra de no crónicos $(\mathrm{z}=-1,448$; $\mathrm{p}=0,148)$.

\section{Calidad de Vida y Apoyo Social}

A nivel del apoyo social objetivo, tal como se puede apreciar en la Tabla 1, éste no correlaciona, como se podía esperar con la $\mathrm{CV}$ en ninguno de los niveles de análisis, a diferencia del apoyo social subjetivo, que solo correlaciona a nivel de la muestra total $\left(r=0,175 ; p=0,007 ; r^{2}=0,031\right)$ de manera directa y con una baja intensidad.

\section{Calidad de Vida y Balanza de Afectos}

Con relación a la balanza de afectos, esta correlaciona con la $\mathrm{CV}$ en todos los niveles de análisis, tanto a nivel general $\left(\mathrm{r}=0,452 ; \mathrm{p}<0,001 ; \mathrm{r}^{2}=0,204\right)$, como por condición (crónicos: $\mathrm{r}=0,432 ; \mathrm{p}<0,001 ; \mathrm{r}^{2}=0,187$; no crónicos: $\left.\mathrm{r}=0,427 ; \mathrm{p}<0,001 ; \mathrm{r}^{2}=0,182\right)$.

De igual manera, el afecto positivo correlaciona significativamente con todos los niveles (total: $r=0,443 ; p<0,001$; $r^{2}=0,196$; crónicos: $r=0,476 ; p<0,001 ; r^{2}=0,227$; no crónicos: $\left.r=0,407 ; p<0,001 ; r^{2}=0,166\right)$. En todos estos casos, la relación es de tipo directa y de baja intensidad.

Al analizar el afecto negativo, se encuentra que éste correlaciona también con $\mathrm{CV}$ a nivel general $(\mathrm{r}=-0,309$; $\left.p<0,001 ; r^{2}=0,095\right)$ y por condición (crónicos: $r=-0,249$; $\mathrm{p}=0,0062 ; \mathrm{r}^{2}=0,06 ;$ no crónicos: $\mathrm{r}=-0,293 ; \mathrm{p}=0,001$; $\left.r^{2}=0,086\right)$, sin embargo, la relación encontrada es de tipo inversa y con una medida del tamaño del efecto que indica una baja intensidad de la relación.

\section{Calidad de Vida y Autoestima}

A nivel de la muestra total, esta relación es estadísticamente significativa, de tipo inversa y de baja intensidad $\left(\mathrm{r}=-0,257 ; \mathrm{p}<0,001 ; \mathrm{r}^{2}=0,066\right)$. Al estratificar el análisis por condición, esta también es significativa tanto a nivel de crónicos $\left(\mathrm{r}=-0,211 ; \mathrm{p}=0,022 ; \mathrm{r}^{2}=0,045\right)$, como de controles $\left(r=-0,279 ; p=0,002 ; r^{2}=0,078\right)$ de manera inversa $y$ con una baja intensidad de la relación. Cabe destacar que dada la puntuación del cuestionario, aunque la relación sea inversa, a mayor autoestima mayor será la calidad de vida reportada.

\section{Calidad de Vida y Variables específicas población crónica}

Al analizar específicamente la población de crónicos por tipo de patología, se puede observar que el grupo de patologías cardiovasculares es el que presenta un mayor porcentaje de personas que evalúan de manera negativa su $\mathrm{CV}$, a diferencia de las patologías de tipo endocrina, que en casi un $28 \%$ de los casos la evalúan como buena (ver Tabla 2).

Pese a esto, al evaluar la diferencia existente entre los rangos promedios entre los grupos cardiovascular, endocrino y músculo -esquelético a través de la prueba no paramétrica $\mathrm{H}$ de Kruskal-Wallis, se encuentra que estas diferencias no son significativas $(\chi 2=3,777 ;$ g.l. $=2 ; p=0,151)$

No se encuentra significativa la relación entre el tiempo de evolución de la enfermedad y la CV.

Sobre la relación existente entre el control percibido de la enfermedad y la CV, se encuentra que esta es estadísticamente significativa de manera inversa y con baja intensidad $\left(\mathrm{r}=-0,283 ; \mathrm{p}=0,002 ; \mathrm{r}^{2}=0,080\right)$, pero dado que las respuestas en esta categoría abarcan desde siempre a nunca, por lo que a mayor puntuación menor control, lo que indica que la relación correcta es a mayor control, mayor calidad de vida.

Al analizar la relación existente entre la efectividad percibida de la medicación y la $\mathrm{CV}$, se encuentra que esta es significativa e inversa $\left(r=-0,283 ; p=0,002 ; r^{2}=0,080\right)$, pero dado que la escala evalúa en categorías desde muy efectiva hasta inefectiva, la interpretación correcta es que a mayor efectividad percibida de la medicación es mayor la $\mathrm{CV}$.

La relación existente entre la percepción de gravedad de la enfermedad y la $\mathrm{CV}$, es significativa y directa $(\mathrm{r}=0,237$; $\left.\mathrm{p}=0,009 ; \mathrm{r}^{2}=0,056\right)$, al igual que la relación existente con la percepción de gravedad de los síntomas $(\mathrm{r}=0,380 ; \mathrm{p}<0,001$; $\left.\mathrm{r}^{2}=0,144\right)$. 
Tabla 2.

Calidad de Vida por Grupo de patologías

\begin{tabular}{lcccccccc}
\hline & \multicolumn{2}{c}{ Mala } & \multicolumn{2}{c}{ Normal } & \multicolumn{2}{c}{ Buena } & \multicolumn{2}{c}{ Total } \\
& frec & $\%$ & frec & $\%$ & frec & $\%$ & frec & $\%$ \\
\hline Tr.Musculoesqueletico & 3 & 23,1 & 8 & 61,5 & 2 & 15,4 & 13 & 100 \\
Tr. Cardiovascular & 15 & 25,9 & 32 & 55,2 & 11 & 19,0 & 58 & 100 \\
Tr. Endocrino & 8 & 24,2 & 16 & 48,5 & 9 & 27,3 & 33 & 100 \\
\hline
\end{tabular}

Frec: frecuencia; \%: Porcentaje

\section{Discusión}

La evidencia encontrada permite sugerir que a pesar de que la gran mayoría de los encuestados, tanto a nivel de crónicos como de no crónicos, evalúa su nivel de $\mathrm{CV}$ como normal, existen diferencias significativas entre ambos grupos, encontrándose que efectivamente el grupo de crónicos relata una menor $\mathrm{CV}$ que el grupo de no crónicos, lo que refuerza la idea de que la presencia de una enfermedad crónica afecta la evaluación de la $\mathrm{CV}$ de una persona.

Existen además otros factores que los resultados indican están relacionados con esta evaluación de la $\mathrm{CV}$.

$\mathrm{Al}$ analizar la relación entre CV y edad, esta es significativa solamente en la muestra de crónicos, pero con una muy baja intensidad de la relación. Dado que tal hallazgo no es reportado de igual manera para el grupo de no crónicos, no podría decirse en términos generales que a mayor edad menor CV (considerando como argumento que avale esta opción que a medida que pasan los años hay un deterioro progresivo de la salud), ni que a menor edad es menor la $\mathrm{CV}$ (tomando como argumento que a medida que pasan los años es mayor el proceso de adaptación de las personas), sino la evidencia encontrada sugiere que la edad incide como un factor modulador de la CV solo en presencia de una patología crónica.

Este hallazgo reafirma la gran mayoría de las investigaciones que han reportado que la edad no es una variable influyente en la CV, sin embargo, en patologías específicas, algunos autores han encontrado alguna relación, como por ejemplo, menor $\mathrm{CV}$ en mujeres con diabetes de mayor edad (Mata, 2003), peor CV en pacientes más jóvenes viviendo con VIH (Swindells, 1999), personas con asma (Mészáros et al., 2005), pacientes dializados (Pucheu et al., 2004) o con las dimensiones física y social en el caso de la edad y sexo para personas viviendo con Parkinson (Frazier, 2000).

Pudiese pensarse que más que el paso del tiempo propiamente tal, pudiese ser el paso del tiempo viviendo con la enfermedad, sin embargo, no se encontró relación significativa entre el tiempo viviendo con la enfermedad y la $\mathrm{CV}$ reportada.

A diferencia de lo que puede pasar con enfermedades de carácter crónico con una evolución degenerativa, como el Parkinson (Frazier, 2000), o lo reportado para una hipertensión de más de 10 años (Dafó et al., 2000) las enfermedades prevalentes en la muestra parecieran no estar mediadas por el paso del tiempo, tal como lo ha sugerido Carroll (1999) para las personas con daño espinal o Pucheu et al. (2004) para pacientes dializados. Esto argumentaría en contra de la opinión de que a mayor tiempo de evolución habría una mayor adaptación a la enfermedad y consecuentemente una mejor calidad de vida.

En este sentido, más que el tiempo que la persona declara vivir con la enfermedad, podría indagarse más en el grado de adaptación y de aceptación de la enfermedad, que probablemente no esté vinculado directamente al paso del tiempo sino a otras variables

Dado el carácter transversal de la investigación, no es posible seguir la evolución de esta percepción en patologías que pudiesen tener un pronóstico de mala evolución, especialmente de tipo físico degenerativas (como las del grupo músculo esqueléticas), existiendo solo referencias de estudios prospectivos en niños y adolescentes con enfermedades crónicas (Sawyer et al., 2004).

Sería interesante además profundizar en futuras investigaciones en la relación existente entre los procesos de aceptación de la enfermedad y la CV percibida, pudiendo ser el caso de que no es el tiempo con la persona vive con la enfermedad un factor decisivo, sino el estado de aceptación que tiene de ésta, ya que en algunas investigaciones se ha encontrado una menor $\mathrm{CV}$ cuando la primera sintomatología aun es reciente (Avis et al., 1996) o lo propuesto por Cummins (2000), que sugiere que la gente tiende a mantener un cierto nivel de bienestar, resguardándose de los cambios ambientales, y sólo cuando estos cambios exceden un cierto límite afectan el bienestar subjetivo de las personas.

Con relación al sexo, los resultados son más concluyentes: el sexo de las personas no es una variable influyente en la CV.

Para algunos autores (Eckermann, 2000), el hecho de que el sexo no sea significativo en la percepción de la $\mathrm{CV}$ guarda relación con que las investigaciones no reflejan las experiencias objetivas y subjetivas de la mujer en salud, enfermedad y discapacidad, pensándose que la experiencia de salud es neutral al género, sugiriendo deconstruir el concepto de lo normal, ya que históricamente se ha vinculado a determinados experiencias de salud (vivencias de síntomas 
sin claro diagnóstico, por ejemplo) a la mujer, creando estereotipos. Otros autores (Mercier, 1998; Pucheu et al., 2004) plantean simplemente que no existirían relaciones significativas entre sexo y $\mathrm{CV}$ percibida, ya que la evidencia empírica así lo demuestra, tal como ha sido el caso de esta investigación.

En nuestro trabajo hemos hallado una relación significativa entre CV general y satisfacción con el estado de salud, tanto al evaluar por país como por condición de morbilidad, lo cual evidencia la influencia de ésta (desde el componente subjetivo de satisfacción) lo que coincide con otras investigaciones (Míchalos, 2000; Avis et al., 1996; Han et al., 2003).

En nuestra investigación se ha encontrado que la media del rango de CV es mayor en aquellos en que prevalece un locus de control interno por sobre el externo, siendo necesario profundizar la investigación de explicaciones, de carácter cognitivo probablemente, que vinculen de alguna manera la CV con la percepción de control, partiendo de la base que aquellas personas que creen que su salud esta mediada por sus propias conductas tienen una mejor evaluación que aquellas que conciben este proceso con una mayor influencia de elementos externos (ejemplo medicación o asistencia sanitaria). Este hecho ha sido reportado por otros autores (Carroll, 1999; Burckhardt, 1985, en Míchalos, 2000; Pucheu et al., 2004) quienes han sugerido la relación entre locus de control en salud y CV (mayor control o control interno estaría asociado a una mayor CV).

Con relación al apoyo social, se encuentra que la $\mathrm{CV}$ se halla vinculada solamente al apoyo social subjetivo a nivel de la muestra total, perdiéndose esta significación al analizar por condición de morbilidad, a diferencia de lo reportado por Han et al. (2003) para población con patología crónica o en mujeres (Achat, 1998), por lo que los resultados en esta área no son concluyentes.

Posiblemente, el tipo de significación que se otorga al apoyo social subjetivo, así como la importancia atribuida a este, en la muestra total, pueda explicar porque solamente este se relaciona con la $\mathrm{CV}$, tornándose más importante el sentimiento de saber que se tiene apoyo (vinculado posiblemente a una mayor importancia a valores de pertenencia, colectivismo y cooperativismo), más que la presencia real de este apoyo.

En el análisis por grupo de morbilidad, ambos tipos de apoyo no se evidencian como influyentes en la CV de población afectada por una patología crónica.

Esta ausencia de vinculación pudiese hacernos pensar que el vínculo apoyo social y CV es independiente de la presencia o ausencia de una enfermedad, aun cuando el sentido común nos lleva a pensar que una persona con una enfermedad para toda la vida percibiría que tiene una mayor CV si sintiese que tiene apoyo y que puede contar con él, hecho que ha sido reportado en algunas investigaciones con patologías crónicas (Ethgen et al., 2004; Wilson \& Cleary, 1995).

A nivel de afectividad, se encuentra relación entre la CV y el afecto positivo (de manera directa), el afecto negativo (de manera inversa) y la balanza de afectos (de manera directa). Este hecho ha sido también reportado en diversas investigaciones, evidenciando la influencia que tiene el equilibrio afectivo en la calidad de vida de las personas (no solo en las afectadas por una enfermedad), llegando algunos autores a plantear este componente como una medida más de calidad de vida, fundamentalmente en el área subjetiva, estableciéndola como un componente básico de la felicidad (Veenhoven, 2000).

De igual manera, en todos los niveles de análisis efectuados en esta investigación, la satisfacción con la vida correlaciona con la $\mathrm{CV}$, siendo también propuesta como un indicador subjetivo de CV dada precisamente, esta alta correlación entre ambas (Veenhoven, 2000).

Al considerar específicamente las variables que afectan a la población crónica, no se encontraron diferencias entre las diversas patologías, pese a que se esperaba que las personas con una patología crónica de tipo degenerativo (Tr. Músculo-esqueléticos, por ejemplo), tuviesen una menor CV con relación a los otros grupos de trastornos estudiados (cardiovasculares y endocrinos), hecho que ha sido reportado por algunos autores (Arnold, 2004; Stavem et al., 2001; Spranger et al., 2000)

Este hecho puede tener su explicación en que los sujetos de la muestra están bajo control médico regular, por lo que probablemente la sintomatología sea poco disfuncional.

Con relación a la sintomatología, cabe destacar que en el caso de las patologías crónicas la evaluación subjetiva tiene su base principalmente en el funcionamiento psicológico y en el grado de disfunción física.

En el caso de esta muestra, la mayoría de patologías estudiadas no ocasionan disfunción grave ni deterioro psicológico cuando están controladas, y aún cuando se pudiese esperar un mayor porcentaje de pacientes con trastornos músculo esquelético con una menor calidad percibida de vida, dado el daño funcional que generan, no se observaron diferencias significativas con los otros grupos de trastornos, pudiendo ser la causa de este hecho el que los casos más graves o con evidente deterioro no sean atendidos en Atención Primaria y concurran a centros de prevención secundaria o terciaria.

Puede que dada la baja gravedad de los síntomas en nuestra muestra, así como el buen control de los mismos bajo una correcta medicación, tengan un grado de influencia en este dato, ya que algunos autores han encontrado vinculación entre esta variable y la CV (Mészáros et al., 2005; Frazier, 2000). En este sentido, para Arnold et al. (2004), las diferencias entre enfermedades pueden deberse a que diferentes dominios específicos son afectados de forma 
diferente en cada enfermedad y por ende, la contribución de cada dominio específico a la calidad de vida general también varía.

Coincidiendo con el hallazgo de algunos autores (Croyle et al., 2003; Burckhardt, 1985, en Michalos et al., 2000; Miller, 1992, en Wahl et al., 1999; Kohler et al., 2002; Deenen, 1993, en Hesselink et al., 2004) nosotros hemos encontrado una relación significativa entre el control percibido de la enfermedad y la CV; esto es, a mayor percepción de control de su enfermedad, mayor calidad de vida, presumiblemente esta vinculación pueda tener un contexto cultural o bien algunos elementos externos estén influyendo en este sentimiento de control, como por ejemplo el manejo clínico, la severidad de los síntomas o la efectividad de la medicación.

Lamentablemente, dado el número insuficiente de casos como para realizar análisis diferenciando por patologías, no se pueda concluir sobre patologías específicas, donde se esperaría comportamientos diferenciales, debido, por ejemplo, a que el grupo de pacientes diabéticos, por las características particulares de la sintomatología y de su tratamiento, en donde el paciente sabe con certeza que a través de la medicación puede controlar completamente los síntomas, relate un mejor control que el grupo con trastornos músculo -esqueléticos, en donde los medicamentos pudiesen en no responder a las expectativas del paciente.

Este último hecho cobra importancia dada la relación encontrada entre la efectividad de la medicación y la calidad de vida en la muestra estudiada, ya reportada por otros autores como Frazier (2000), en donde en todos los niveles de análisis, excepto los enfermos músculo-esqueléticos, existe un relación significativa entre la efectividad de la medicación y la calidad de vida. Esto puede tener su explicación en las características del tratamiento y la medicación, la cual puede ser tan efectiva que deja de convertirse en un elemento consciente vinculado a la calidad de vida general.

Con relación a la percepción de gravedad de la enfermedad y a la gravedad de los síntomas, se encuentra una relación significativa entre ésta y la calidad de vida. Probablemente influyan en esta variable (la percepción de gravedad) el grado de información sobre la propia enfermedad y el grado de controlabilidad (si se puede controlar es menos grave), lo que a su vez repercutiría en el bienestar subjetivo y este a su vez en la evaluación global de calidad de vida.

En este sentido sería interesante comprobar lo que sucede en pacientes con distintos grados de gravedad de una misma patología, en patologías terminales o con difícil manejo de la sintomatología y en pacientes con baja adhesividad al tratamiento, las que pudiesen ser variables que influyesen en la posible relación locus de control-calidad de vida.

Se considera necesario, además de ampliar la muestra en futuras investigaciones, evaluar el nivel de impacto de cada una de las variables estudiadas en cada uno de los diversos dominios que componen la $\mathrm{CV}$, así como el análisis diferenciado por patología, lo que permitirá mayor certeza a la hora de planificar intervenciones destinadas a mejorar la CV de los pacientes viviendo con una patología crónica.

\section{Referencias}

Achat, H., Kawachi, S., Levine, S., Berkey, C., Coakley, E. \& Colditz, G., (1998) Social Networks, stress and health related quality of life. Quality of life research. 7, $735-750$.

Alonso, J., Ferrer, M., Gandek, B., Ware, J., Aaronson, N., Mosconi, P., Niels, K., Rasmussen, K., Bullinger, M., Fukuhara, S., Kaasa, S., Lelége, A., \& IQOLA Project Group (2004). Health related quality of life associated with chronic conditions in eight countries: Results from the international quality of life assessment (IQOLA) project. Quality of life research, 13, $283-298$.

Arnold. R., Ranchor, A., Sanderman, R., Kempen, G., Ormel, J. \& Suurmeijer, T. (2004). The relative contribution of domains of quality of life to overall quality of life for different chronic diseases. Quality of life research, 13, 883-896.

Avis. N., Smith, K., Hambleton, R., Feldman, H., Selwyn, A. \& Jacobs, A., (1996). Development of the multidimensional Index of life quality: a quality of life measure for cardiovascular disease. Medical Care, 34, $1102-1120$.

Barros da Silva, A., Fleck, M., Pechansky, F., de Boni, R. \& Sukop, P., (2005). Psychometric properties OF THE World Health Organization Quality of Life instrument (WHOQoL-BREF) in alcoholic males: A pilot study. Quality of Life Research. 14:473-478.

Bennet, S., Perkins, S., Lane, K., Deer, M., Brater, D. \& Murray, M. Social Support and health related quality of life in chronic heart failure patients. Quality of life research, 10, 671-682.

Carroll,A., (1999) The relationship between control beliefs, coping strategies, time since injury and quality of life among persons with spinal cord injuries (abstract). Dissertation Abstracts International: Section B: The Sciences and Engineering, 60(6-B), 2934.

Cassileth, B. R., Lusk, E. J., Strouse, T. B., Miller, D. S., Brown, L. L., Cross, P. A. \& Tenaglia, B. S. (1984). Psychosocial status in chronic illness; a comparative analysis of six diagnostic groups. The New England Journal of Medicine, 23, 506-511.

Croyle,K., Troester,A., Fields,J., Straits-Troester,K., Lyons,K. \& Pahwa,R., (2003). Presurgical coping, depression, and quality of life in persons with Parkinson's disease. Journal of Clinical Psychology in Medical Settings, 10(2), 101-107.

Cummins, R.A. (2000). Objective and subjective quality of life: an interactive model. Social Indicators Research, 52, 55-72

Dalfó A., Badia, X., Roca, A., Aristegui, I. \& Roset, M., (2000). Validación del cuestionario de calidad de vida en hipertensión arterial (CHAL) para su uso en España. Relación entre variables clínicas y calidad de vida. Atención Primaria, 26 (2), 70 - 85.

Eckermann, L. (2000). Gendering indicators of health and well being: is quality of life gender neutral? Social Indicators Research, 52, 29 -54 .

Ethgen, O., Vanparijs, p., Delhalle, S., Rosant, S., Bruyere, O. \& Reginster, J. (2004). Social Support and health related quality of life in hip and knee osteoarthritis. Quality of life Research, 13, 321 - 330.

Frazier,L., (2000) Coping with disease-related stressors in Parkinson's disease. Gerontologist, 40(1), 53-63.

Han, K., Lee, P. \& Park, E., (2003). Factors influencing quality of life in people with chronic illness in Korea. Journal of nursing scholarship, 35(2) $139-144$.

Hart, H., Bilo, H., Redekop, W., Stolk, R., Assink, J. \& Meyboom de Jong, B., (2003) Quality of life of patients with type I diabetes mellitus. Quality of lfe research, 12, 1089 - 1097.

Hesselink, A., Penninx, B., Schlösser, M., Winjhoven, H., van der Windt, D., Kriegsman, D. \& van Ejik, T., (2004).The role of coping resources 
and coping style in quality of life of patients with asthma or COPD Quality of life Research, 13, 508-518.

Jang, Y., Hsieh, C., Wang, Y. \& Wu, Y., (2004). A Validity study of the WHOQoL-BREF Assessment in persons with traumatic spinal cord injury. Arch Phys Med Rehabilitation, 85, 1890-1895.

Kempen, G., Jelicic, M. \& Ormel, J., (1997). Personality, Chronic Medical Morbidity, and health related Quality of life among Older Persons. Health Psychology, 16 (6), 539 - 546.

Kohler, C., Fish, L. \& Greene, P., (2002).The relation of perceived selfefficacy to quality of life in chronic Obstructive Pulmonary Disease. Health Psychology, 21 (6), 610 - 614.

Lucas C., R., (1998). Versión Española del WHOQoL. División de Salud Mental. Organización Mundial de la Salud. Ed. Ergon S.A., Madrid.

Luscombe, F., (2000). Health related quality of life and associated psychosocial factors in irritable bowel syndrome: A review. Quality of Life Research, 9, $161-176$.

Mata, M., Roset, M., Badía, X., Antoñanzas, F. \& Alcázar, J., (2003). Impacto de la diabetes mellitus tipo II en la calidad de vida de los pacientes tratados en las consultas de atención primaria de España. Atención Primaria, 31 (8), 493 - 499.

Mercier, C., Péladeau, N. \& Tempier, R., (1998). Age, gender and quality of life. Community Mental Health Journal, 34 (5), 487 - 500.

Mészáros, A., Zelkó, R., Meskó, A. \& Vincze, Z. (2005). Factorial desig for the analysis of patient's quality of life in asthma. Quality of life Research, 14, $191-195$.

Michalos, A., Zumbo, B. \& Hubley, A., (2000). Health and the quality of life. Social Indicators research, 51, $245-286$.

Pucheu, S., Consoli, S., D Auzac C., Francais, P. \& Issad, B. (2004). Do Health causal attributions and coping strategies act as moderators of quality of life in peritoneal dialysis patients? Journal of Psychosomatic Research, 56, $317-322$.

Rudnick, A., (2001). The Impact of Coping on the relation between symptoms and Quality of life in Schizofrenia. Psychiatry, 64 (4), $304-308$.

Sawyer, M., Reynolds, K., Couper, J., French, D., Kennedy, J., Staugas, R., Ziaian, T. \& Baghurst, P. (2004). Health related quality of life of childrens and adolescents with chronic illness - a two year prospective study. Quality of life Research, 13, 1309 - 1319.
Saxena, S., Carlson, D., Billington, R. \& Orley, J.(2001). The WHO quality of life assessment instrument (WHOQOL- Bref): The importance of its items for cross-cultural research. Quality of life Research, 10, $711-721$

Skevington, S., Lotfy, M. \& O'Conell, K., (2004) The World Health Organization's WHOQOL-BREF quality of life assessment: Psychometric properties and results of the international field trial A report from the WHOQOL Group. Quality of life Research, 13, 299-310.

Spranger, M., de Regt, E., Andries, F., van Agt,H., Bijl, R., de Boer, J., Foets, M., Hoeymans, N., Jacobs, A., Kempen, G., Miedema, M., Tijhuis, M. \& de Haes, H., (2000). Which chronic conditions are associated with better or poorer quality of life? Journal of Clinical Epidemiology, 53, 895- 907.

Stavem, K., Lossius, M., Kvien, T. \& Guldvog, B., (2001). The health related quality of life of patients' with epilepsy compared with angina pectoris, rheumatoid arthritis, asthma and chronic obstructive pulmonary disease. Quality of life, 9, $865-871$.

Swindells, S., Mohr, J., Justis, J., Berman, S., Squier, C., Wagener, M. \& Singh, N., (1999). Quality of life in patients with human immunodeficiency virus infection: Impact of social support, coping style and hopelessness. International Journal of STD and AIDS, 10(6), 383-391.

WHOQOL Group (1998). Development of the World Health Organization WHOQOL-BREF Quality of Life Assessment. Psychological Medicine, $28,551-558$

Veenhoven, R. (2000) The Four Qualities of life. Ordering concepts and measures of the good life. Journal Of Happiness Studies, 1, 1-39.

Wahl, A., Hanestad,B., Wiklund,I. \& Moum,T., (1999). Coping and quality of life in patients with psoriasis. Quality of Life Research, $8(5), 427-433$

Wallston, K.(1999) Multidimensional Health Locus of Control (MHLC) Scales. Extraído el 15 de enero de 2005 del sitio web de la Universidad de Vanderbilt: http:/www.vanderbilt.edu/nursing/kwallston/ mhlcscales.htm

Wilson, I. \& Cleary, P., (1995). Linking clinical variables with health related quality of life. Journal of American Medical AssociationJAMA, 273 (1), 59 - 65. 